\title{
THE STRUCTURE OF ROUND STELLAR SYSTEMS: OBSERVATION AND THEORY
}

\author{
IVAN R. KING \\ Dept. of Astronomy, University of California, Berkeley, Calif. 94720, U.S.A.
}

\begin{abstract}
In addition to its traditional roles of checking theories and applying them, observation also plays an important role in guiding theory and indicating the form that it should take. This paper reviews types of observation, types of stellar system (omitting the highly flattened ones), and the relations between them. Observational limitations are indicated, as well as places where observations of particular sorts are needed, either for guidance of theories or for their application.
\end{abstract}

\section{Introduction}

This review will attempt to assess the role of observations of spherical and moderately flattened stellar systems, from the point of view of theory. The main concern will be with two questions: what can observations do for theory, and what do observations say about theories? Some discussion will also be given of how observations can be used to apply theory, in order to derive significant astronomical results.

In principle, observations can assist the theoretician in several different ways. First, they can act as a general guide for the theory, indicating the directions in which it should go. The fortunate cases are very few in which a theory can start completely a priori, idealizing the problem in a reasonable way and proceeding to a correct theoretical picture. More often, the theoretical problem lacks clear definition, and we must first look at the Universe to see what the significant conditions and parameters are. An example of this situation is the dynamics of globular clusters, where the observation of a tidal limit, in the very first star count made on a Palomar Schmidt plate, showed that the tidal cutoff played a vital role in the dynamical problem (King, 1962). The second major way in which observations can contribute to theory is to discriminate between various theoretical possibilities, each of which predicts different observational consequences. We shall see an example of this below, in the attempt to distinguish between various possible cutoffs in a velocity distribution. Later on, of course, there is the task of testing a fully developed theory, to see whether its predictions are in agreement with observation. Finally, when a theory is accepted as being valid, observational data are needed in order to apply it, to reach concrete conclusions about the Universe.

In principle, we can observe almost everything about the state of a stellar system - at least at the present moment of time - but in practice there are many difficulties and limitations. We can observe two of the three position coordinates, lacking only the line-of-sight component, and in principle we can observe all three components of the velocity of each star, by combining radial velocities and proper motions. In practice, however, it is almost never possible to derive such detailed information, at least with accuracy that has any practical significance. 
Accuracy is of course a serious problem in any set of observations, but this fact is not always as obvious as it should be. There are sets of observations in the literature that are afflicted with serious errors. This is not to say that all observations should be rejected, or regarded as suspicious; but one must always have an eye to the quantitative reliability of the observations that are in hand.

Even when the accuracy is completely adequate, observations of the structure of stellar systems are limited by three fundamental problems. The first problem is faintness: we need to observe the number and distribution of faint stars, the brightness distribution of the integrated light in faint envelopes, and radial velocities from faint spectra. In every case the faintness problem limits both the quantity and the quality of the observations, which often stop tantalizingly short of the interesting level. The second problem is background. The dynamical understanding of stellar systems often depends heavily on knowing the density distribution in their outer envelopes, and here the background is nearly always a limitation. Statistical fluctuations in the number of background stars limit the distance to which we can detect the outer envelopes of globular clusters, and this interference makes it difficult to study the envelopes of open clusters at all. For clusters of galaxies the problem is even worse, because of clustering of the background galaxies. Finally, the outer envelopes of elliptical galaxies are lost in the light of the night sky. The third problem is small numbers. Sometimes this is merely an economic problem: for example, where the limitation of observing time has allowed velocities of only a dozen objects to be measured. Sometimes, however, it is an essential problem: for example, how does one discuss the dependence of distribution on stellar mass, in a cluster that has a total of only 50 stars?

This last question raises another fundamental problem which pervades the interface between observation and theory. Just how are the two to be compared when the observation always provides only a subset of the quantities that exist in a theoretical model ? The answer, I believe, is clear and unequivocal - and very important to follow in practice. The observations, limited as they are in accuracy, should never be transformed into a different set of quantities, for comparison with theoretical predictions. It is rather for the theoretical results to be transformed into the observational domain, for direct comparison with unadulterated observations. Quite aside from the question of whether the theory is correct or completely wrong, it can be carried out to as many significant figures as one desires; hence it does not suffer from statistical errors that are magnified in the transformation. The observations, however, have accidental errors that are nearly always increased when they are transformed to another domain. (Orthogonal transformations, such as the Fourier transform, are the one exception.)

A good example is density distributions, where the observations are made in a two-dimensional domain. Transformation from a projected to a spatial density distribution requires, whatever the method may be, a numerical differentiation of observed densities, which greatly increases the accidental errors. A theoretical distribution in three dimensions, however, can easily be projected into a two-dimensional 
distribution without any loss of accuracy that matters. Particularly insidious is the observational use of strip counts, in which perfectly good two-dimensional densities are degraded into a one-dimensional marginal distribution before being processed further.

\section{Types of Observation}

For the stellar systems considered here, the observations consist mainly of density distributions. Velocity distributions, valuable though they are, are much more difficult to obtain, and consequently much less velocity information is available.

Density distributions may be determined by counting individual stars, or by surface photometry of the integrated light. The data may be registered photographically, photoelectrically, or electrographically. Each technique has its advantages and its disadvantages. The great advantage of direct photography is that a single photograph can take in a very wide area. Not only is this an economic advantage, but it also avoids the sometimes costly errors that are involved in converting separate observations onto the same basis. Its disadvantages are its relatively low photometric accuracy and the difficulty of converting the non-linear response of the photograph into a correct intensity scale.

For accuracy and linearity, photoelectric observations are far superior. (Not all the photoelectric observations in the literature, however, have as high an accuracy as one might hope.) But observations made with conventional photoelectric photometers have two severe disadvantages. First, they are slow and uneconomical, requiring the point-by-point scanning of large areas. Second, they are unable to work at high spatial resolving power. The effects of time-changes in seeing require photoelectric observers to use an aperture that is far larger than the resolution element of a photograph taken under the same conditions. Both these disadvantages are avoided by panoramic detectors, which are coming into increasing use. In fact, because of the inherently higher quantum efficiency of photoelectric surfaces, panoramic detectors are much more efficient than conventional photographs - over the limited area that they cover. For large areas, however, photographs are unmatched, giving as many as $10^{8}$ picture elements, compared with $10^{5}$ for a large panoramic detector.

Electrography is a technique that shares some of the advantages of both houses. It combines the accuracy, linearity, and speed of its photoelectric detector with the resolving power and capacity of its photographic registration. Although the photosurfaces themselves are similar in size to those of panoramic detectors, the fine-grain photographic emulsions that are used in electrography give numbers of picture elements far superior to those of panoramic photoelectric detectors. Electrography has its disadvantages, however. Only a small number of electrographic devices have worked successfully, and the plates introduce special measuring problems, with their sharp resolving power and very high density range.

Each type of stellar system poses its own observational problems and suggests different observational techniques. For open clusters the overriding problem is the small number of stars. Surface-brightness techniques are useless; one must study the 
individual stars. Background numbers are a serious problem, since nearly all open clusters lie in the rich star fields of low galactic latitudes. Fortunately, proper motions are available for individual stars in a number of clusters; with these, the field stars can be weeded out with a high degree of probability. Even so, the star numbers are small. Nevertheless, systems with a small number of stars pose, as we know, a dynamical problem that is essentially different from that of systems with large $N$; and open clusters are the place where we can study such systems observationally.

Globular clusters can be studied by star counts or by surface photometry. The latter technique suffers somewhat from statistical uncertainties, since the integrated light of each region comes from a relatively small number of luminous stars (King, 1966b). In the center of a globular cluster, however, we must resort almost completely to surface photometry, because the individual stars are too crowded to count. Crowding is an even more serious problem than a glance at a photograph would suggest. Comparison of large-scale with small-scale photographs shows that crowding begins to affect star counts as soon as there is one star per 50 or 100 picture elements.

Again here, electrography comes closest to having the best of both worlds. It can reach faint individual stars at the edges of clusters, and it can measure accurate surface brightnesses in the centers. Most important of all, electrographic studies can disentangle crowded and even overlapping star images, because of the high resolution, the linearity, and the large dynamic range.

Even so, globular clusters present a frustrating problem of incomplete observations: we have the distribution of bright stars only at the center and the distribution of faint stars only at the edge. At the center of a typical globular cluster, crowding makes it impossible to study any except the brightest stars. (Surface photometry pushes the effective magnitude limit only a little fainter, since nearly all the light is contributed by the most luminous stars.) Outside the crowded central region, where the fainter stars can be studied, the distribution of bright stars is poorly determined, just because their numbers are so small. Thus no single group can be observed from center to edge, and the observations give only very limited information about one of the most interesting dynamical questions, the relative distribution of stars of different mass. To make this situation even worse, nearly all of the magnitude range that is observed in a globular cluster is inhabited by stars that have evolved from a small part of the original main sequence, around the present-day turnoff; hence these stars all have nearly the same mass. The observations end at a magnitude only a little below the main-sequence turnoff, just where mass is beginning to vary significantly with magnitude.

For elliptical galaxies, where the stars are too faint to detect individually, distributions can be studied only by surface photometry. Near the center, where the brightness is ample, the only serious problem is angular resolving power; at the distances of even nearby ellipticals, core radii are often of the order of a second of arc. Farther out, the eventual limitation is the light of the night sky - or rather its uncertainties and fluctuations; galaxy profiles begin to lose accuracy seriously when the light of the galaxy adds only a few percent to the night sky. 
Density distributions in clusters of galaxies have problems similar to those of open star clusters : first, the numbers are generally small, and second, the envelope of the cluster is too easily lost in the background. Here again, it is extremely valuable to use motion criteria to separate members from background galaxies; in this case the criterion is radial velocity.

Velocities, which are much less available than positional distributions, can be applied in various ways to the study of stellar systems. As already indicated, they can be used to eliminate non-members. Their sizes - more specifically, the velocity dispersion - can be used to determine the mass of the system. The velocity dispersion can be determined from radial velocities of individual objects (or, for a few of the nearest open clusters, from proper motions), and in the high-surface-brightness centers of globular clusters and elliptical galaxies it can be determined from the broadening of lines in the integrated spectrum. Both individual velocities and integrated spectra can be used to study the rotation of a stellar system, whose relation to the velocity dispersion is of great dynamical significance.

The paramount problem in velocity observations is the faintness of the light. It restricts the observations to low spectroscopic dispersion, with a consequent low accuracy. For individual velocities, only luminous stars can be reached, and their numbers are few. For the integrated light, spectra are available only in a small central region of a stellar system. These restrictions leave us with a very limited picture of velocity distributions. Where observations exist at all, they usually provide only a value for the overall velocity dispersion. In a few cases, central angular velocities of rotation are available. Detailed velocity distributions are simply too much to hope for, but it seems possible at least to determine the gross features of some rotation curves, as well as the change of velocity dispersion as a function of radius.

\section{Globular Clusters}

Stellar systems pose theoretical problems of several different sorts, connected respectively with the initial rapid changes; with the nearly-steady condition into which the system then settles, and with the evolutionary processes that change that condition. Although observations do shed indirect light on evolutionary processes, we will concentrate here on the steady phase and its characteristics, because this is the dynamical problem that the observations illuminate most directly. Within this context, it is natural to devote our attention to globular clusters. Not only are they rich enough systems that their density distributions can be determined with a high degree of significance; their dynamical time scales are also short enough that all types of dynamical processes should be important.

On the crudest level, we can ask what kind of density distributions globular clusters have. Observationally, the answer is very simple; a cluster is described by three parameters: the core radius, the limiting radius, and a number factor such as the integrated magnitude. Since the core radius is set independently by the cluster's gravitational binding energy, the limiting radius by the tidal field of the Milky Way, 
and the number factor by its total mass, the absence of further differences shows that globular clusters are as similar as they can possibly be. The dynamical reason is easy enough to see: clusters are more than a relaxation time old, and relaxation has made them what they are.

More generally, the density distribution in a stellar system is our clue to its velocity distribution, which is in turn our clue to its internal dynamics. The velocity and density distributions are rigidly connected by the time-independent Liouville equation (along with Poisson's equation to describe the gravitational field), so that a velocity distribution corresponds to a unique density distribution. Roughly speaking, the low-velocity center of the velocity distribution corresponds to the core of the density distribution, and the high-velocity tail corresponds to the spatial envelope.

Mixing processes - either the initial violent relaxation or the subsequent relaxation through stellar encounters - make the velocity distribution in a stellar system tend toward a Gaussian form. Because the position space of the system is infinite in extent, however, relaxation can never be complete. Deviations from relaxation, and from the corresponding Gaussian velocity distribution, will thus show themselves in the envelope of the system and correspondingly in the tail of the velocity distribution. This means that stellar systems should all have similar cores, while the envelopes can be expected to show the nature of the relaxation process and the way in which it has affected the cluster.

In globular clusters the relaxation comes from stellar encounters; and after the initial settling down, its chief effect is to drive stars across the tidally set boundary of the cluster. Thus the form of the envelope is determined simply by the location of the boundary. Dynamically, however, the limitation is set not by the spatial location of the boundary but rather by the energy that a star needs in order to reach it. Since relaxation drives a steady flow outward through velocity space, the velocity distribution drops smoothly to zero at the cutoff velocity (Spitzer and Härm, 1958); and the corresponding spatial distribution fits the envelopes of globular clusters quite well (King, 1966a). Figures 1 and 2 show examples. If the shape of the cutoff were different, the profile of the cluster's envelope would also be different, as shown in Figure 3. (For extensive collections of globular-cluster data, see King et al., 1968, and Peterson and King, 1975.)

The close agreement shown in Figures 1 and 2 does not mean that we understand the envelope of globular clusters completely, however. The models that produced the curves in Figure 3 were all based on velocity distributions that are everywhere isotropic; yet it is quite possible that the velocities in the outer parts of a stellar system may be quite anisotropic. The theoretical case can be argued either way. On the one hand, the initial formation of a stellar system probably involves a collapse that endows stars with radial motions; furthermore, the ejection of stars from the core into the envelope, by encounters, leaves them with motions that are largely radial. On the other hand, stellar encounters should randomize the angular momenta in the cluster core, and tidal forces should randomize them in the envelope; randomized angular momenta correspond to an isotropic velocity distribution. 


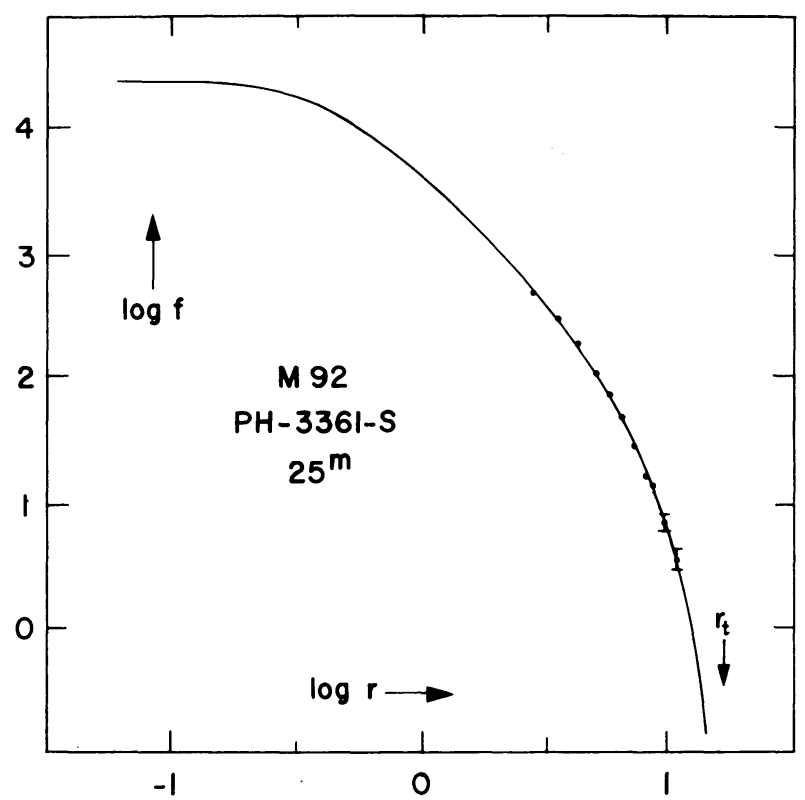

Fig. 1. Fit of observed surface densities in a high-concentration globular cluster. Points represent surface densities, $f$, determined from star counts in rings; vertical bars are statistical uncertainties. Central region was too crowded to count. Curve represents projected densities from a theoretical model with tidal limit at $r_{t}$.

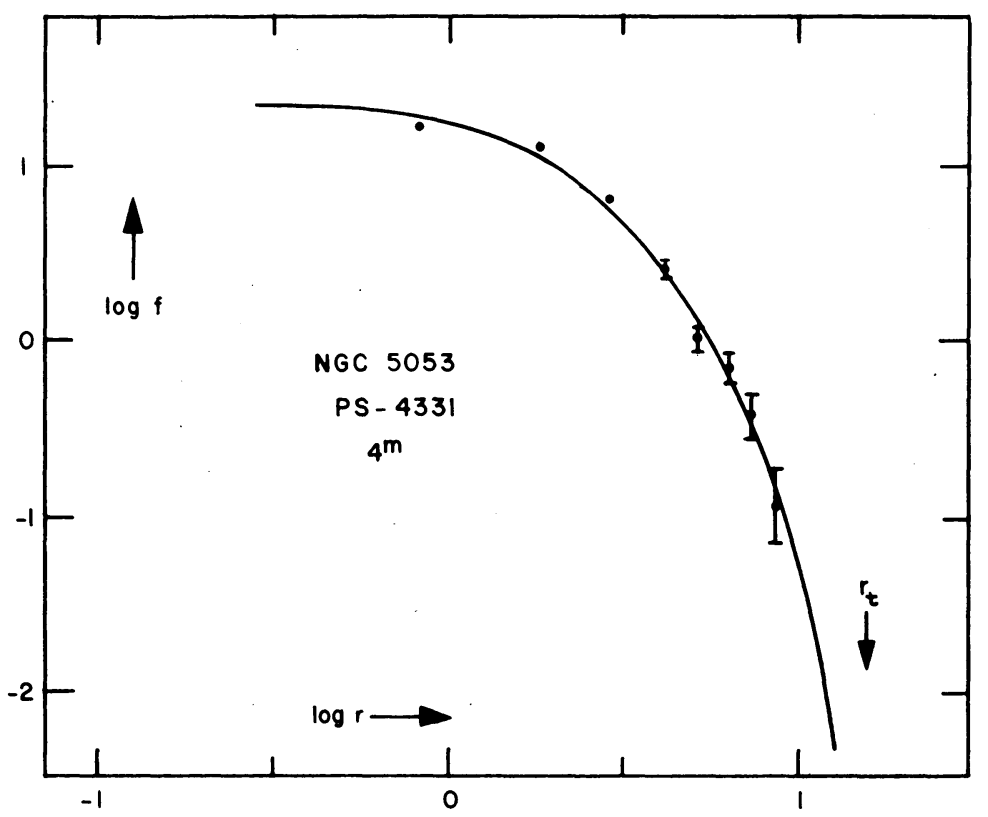

Fig. 2. Fit of observed surface densities in a low-concentration globular cluster. Representation as in Figure 1. 


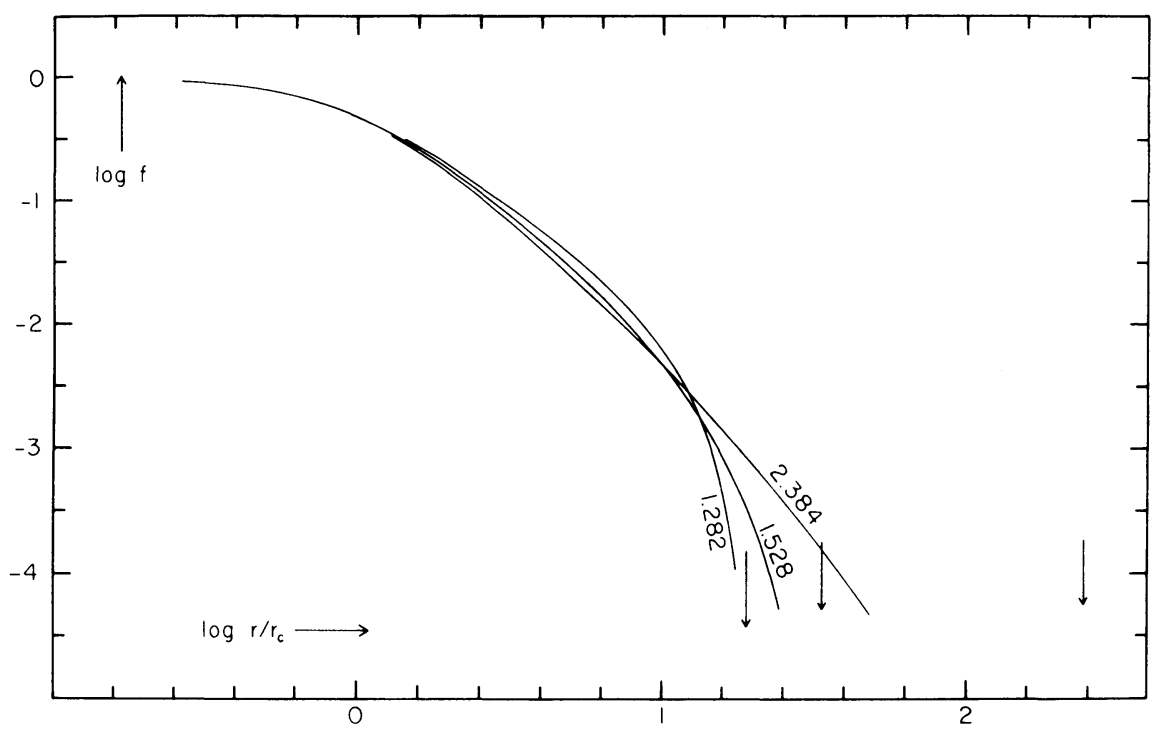

Fig. 3. Projected density vs radius for theoretical models with different velocity cutoffs. Label on each curve is $\log r_{t} / r_{c}$; all have same $r_{c}$. In order of increasing label, models have their velocity distribution truncated at escape velocity $v_{e}$, going to zero linearly as $\left(v-v_{e}\right)$, and going to zero quadratically as $\left(v-v_{e}\right)^{2}$.

Anisotropy in a velocity distribution has its effect on the spatial density distribution, and we may ask whether such effects are observable. Figure 4 shows an example of the effect of anisotropy; for an identical core and inner envelope, the outer envelope of the anisotropic model has a much smoother gradient and a much greater extent. The differences resemble, in an unfortunate way, the effects of changing the shape of the velocity cutoff, and it is thus difficult to distinguish between these two types of phenomenon. There seem to be two ways in which the distinction can in principle be made. First, Figure 4 makes it clear that an anisotropic-velocity envelope has a larger limiting radius than that of the corresponding isotropic model; anisotropic models can, in fact, be designed to have envelopes whose extent is infinite (Oort and van Herk, 1959; Michie and Bodenheimer, 1963). Since we know, from calculations of the galactic tidal field, an upper limit to the distance to which the envelope of any given cluster can extend, this allows us to set a corresponding upper limit on the anisotropy of its velocity distribution.

Probably the best way to study the question of anisotropy would be through measurements of the variation of the line-of-sight velocity dispersion with distance from the center. If the velocities in the envelope are largely radial in direction, they will have only a small component along the line of sight, and the velocity dispersion will drop sharply in the envelope. To interpret such an effect, however, it is essential to use a complete and self-consistent dynamical model of the cluster, since the mere existence of a finite limiting radius will cause the velocity dispersion to fall off radially even when the velocity distribution is isotropic (King, 1966a, Table I; King, 1972 , 


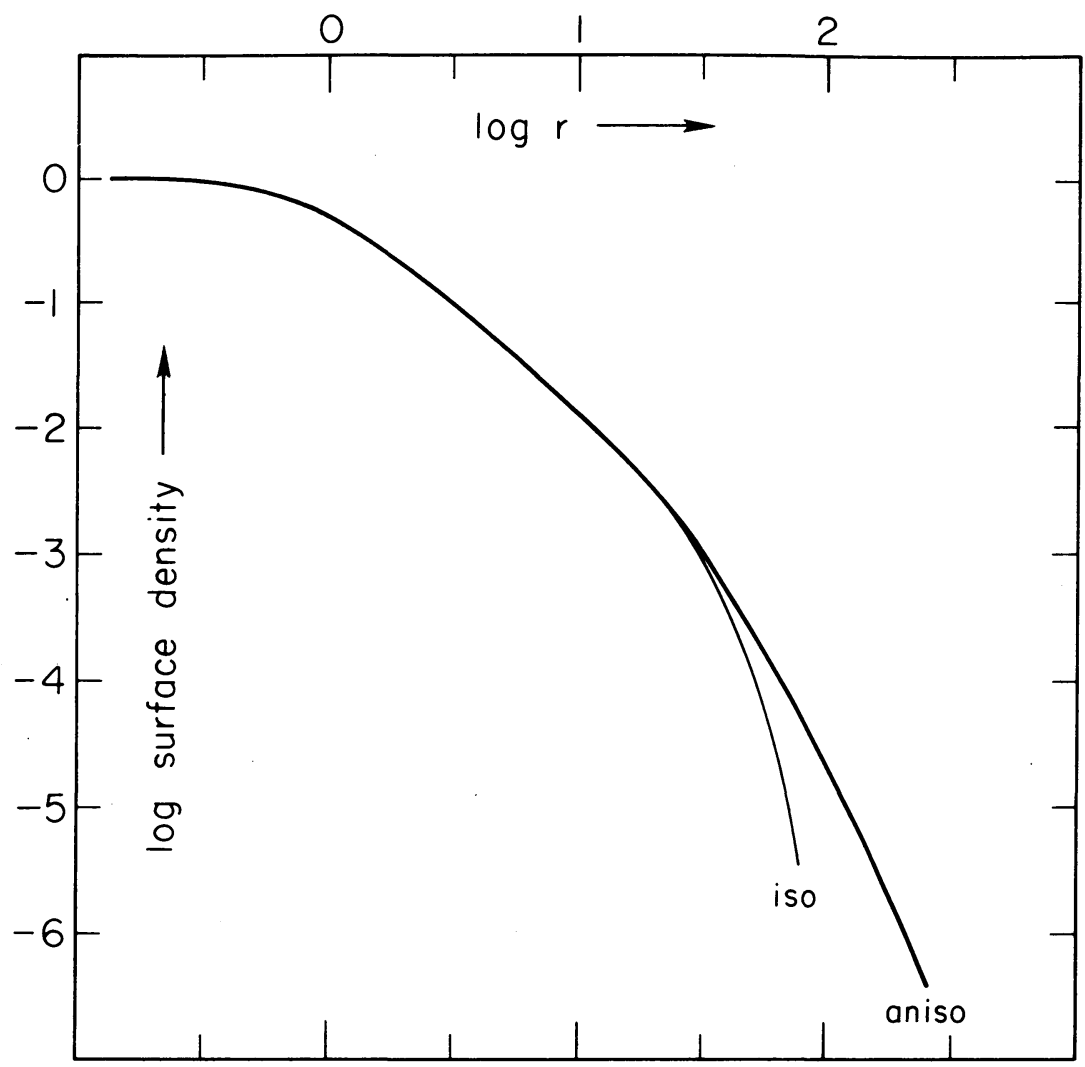

Fig. 4. Projected density vs radius, in two models with same core, to illustrate effect of anisotropy in the velocity distribution.

Table 2). Observations that would settle this question appear to exist (Gunn and Griffin, 1971; Griffin, 1972); when these data are made available to the astronomical community, a long-standing problem will be removed.

Determination of limiting radii of star clusters have other applications. Peterson (1974) has used them to study the shapes of globular-cluster orbits in the Milky Way, arguing that the limiting radius of each cluster shows how close its orbit takes it to the galactic center. Hodge (1966) has made similar calculations for neighboring dwarf elliptical galaxies.

The foregoing discussion has touched on one level of the dynamics of star clusters, but it has not yet distinguished between stars of different mass. Relaxation through stellar encounters is a mechanism that does make a distinction between stellar masses, and systems that have undergone such relaxation should show differences in distribution between stars of high and low mass. Not so for systems that have undergone only an initial violent relaxation, however, since violent relaxation treats all masses equally. Thus star clusters, with their relatively short relaxation times, should show mass segregation, while elliptical galaxies should not. 
Differences in distribution, for stars of different mass, are perversely difficult to determine in globular clusters. As previously indicated, the bright stars can be studied in the core but are too few to give good statistics in the envelope, while the faint stars are well observed in the envelope but cannot be resolved in the core. Only in the globular clusters of lowest central concentration can we observe faint stars in the center, but here our dynamical study is frustrated again. In low-concentration clusters the density distributions are determined much more by the cutoff than by the circumstances in the core, and the distributions of the different stellar types thus look very similar. It is in the theoretical models of highly concentrated clusters that segregation effects are most striking, but there we are unable to observe enough of the center to detect the effects that we are looking for.

This situation is illustrated by Figures 5 and 6, which were plotted from theoretical models in which the stellar masses and the luminosity function are chosen to represent those in a real globular cluster. The first of the figures shows that in a low-concentration cluster the distributions of bright and faint stars are practically indistinguishable. The second figure shows how the differences predicted in a high-concentration cluster elude observation.

There is one situation, however, in which a study of mass segregation has the

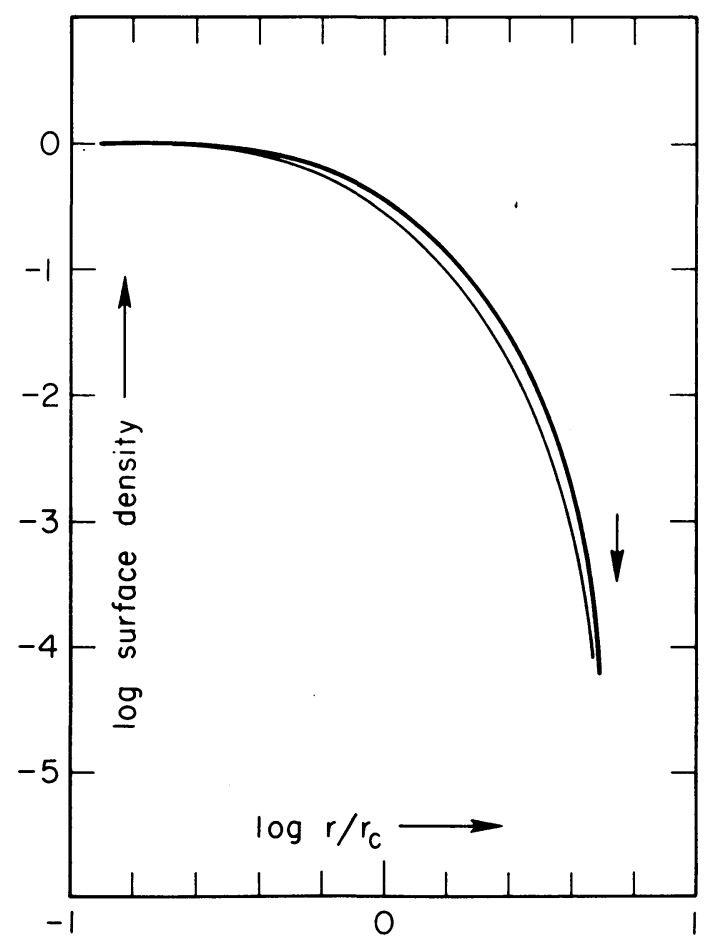

Fig. 5. Surface density vs radius in a low-concentration globular-cluster model. Lower and upper curves refer to stars down to limits $M_{V}=+2$ and +10 , respectively. Central densities have been normalized to same value. 


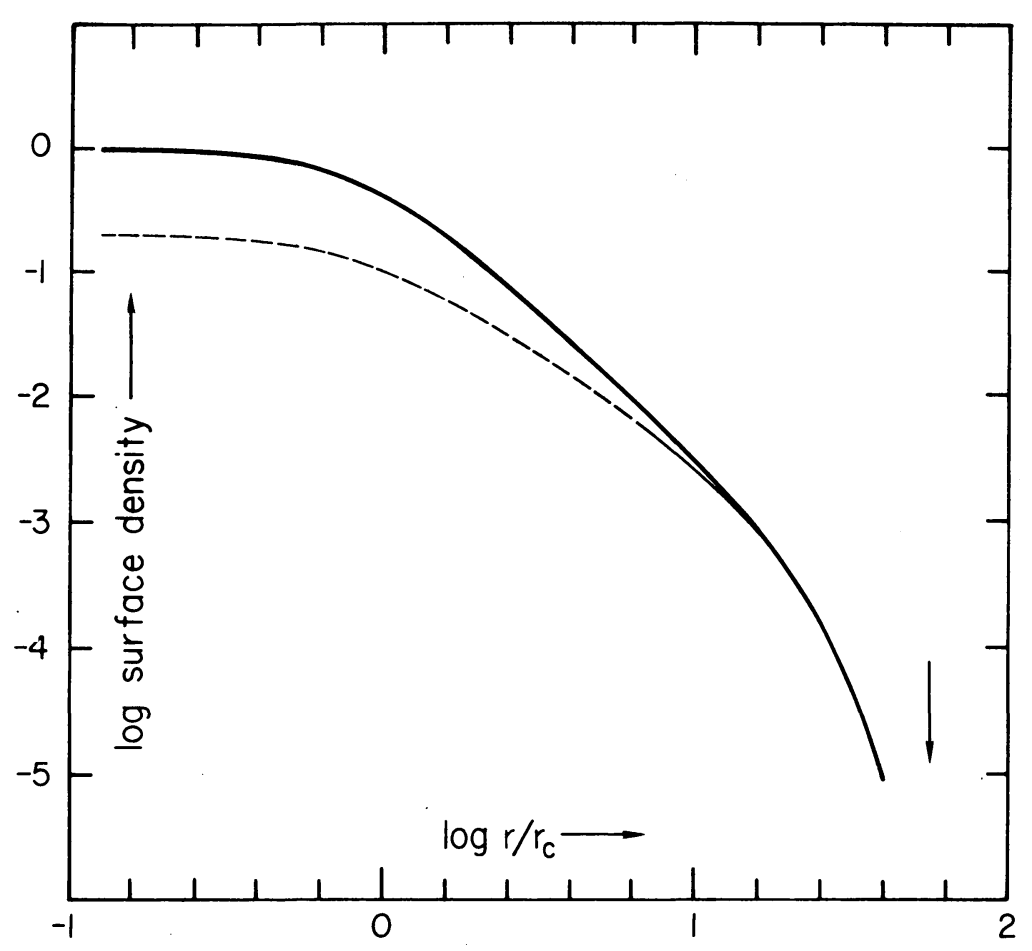

Fig. 6. Surface density vs radius in a high-concentration globular cluster. Upper and lower curves refer to stars down to limits $M_{V}=+2$ and +10 , respectively, but dashed part is unobservable because of crowding. Outer densities have been normalized to same values.

possibility of being successful. If a group among the brighter, evolved stars has lost mass and then relaxed to a higher velocity dispersion, their broader spatial distribution might be observable. The number of stars is small, but these brighter stars can be observed throughout the entire cluster, on short-exposure plates that are bothered very little by crowding effects.

Although such studies can be carried out in principle, in practice I do not know of any observational data that are adequate. I once tried to do a study of this sort, using published counts of stars of various types in concentric annuli. The analysis consisted of comparing the observed numbers with numbers computed for a model in which stars of different types were distributed in accordance with their masses. For each stellar type the low-velocity part of the velocity distribution corresponded to the appropriate Maxwellian distribution, but the high velocities of course had a cutoff of the sort that has already been described. The technique was to use a chi-squared test of goodness of fit, comparing an observed distribution with the computed distribution of stars of each mass. The resulting curve of chi-squared against mass would show a minimum at the mass to which the observed group fitted best. At first the results looked very encouraging: the minimum value of chi-squared corresponded to a good probability level, and in many cases a change of only $20 \%$ in the mass would 
make the fit quite bad. I abandoned the study, however, when it turned out that data from different, but equally credible, sources gave different results. An example is shown in Figure 7 . I believe that the difficulty of determining good stellar distributions in the centers of clusters has been underestimated. Perhaps this is another task for electrography.

A related problem, in which mass segregation plays a role and must be allowed for, is the determination of luminosity functions in globular clusters. While the brighter stars are counted throughout the cluster, the fainter stars can be counted only in an outer annulus, and their total number involves an extrapolation into the center, which can be made correctly only by use of a dynamical model of the cluster. Numbers in an actual case are illustrated in Figure 8. When model fitting is used in this way (Wilson and King, 1975), the previously reported differences between luminosity

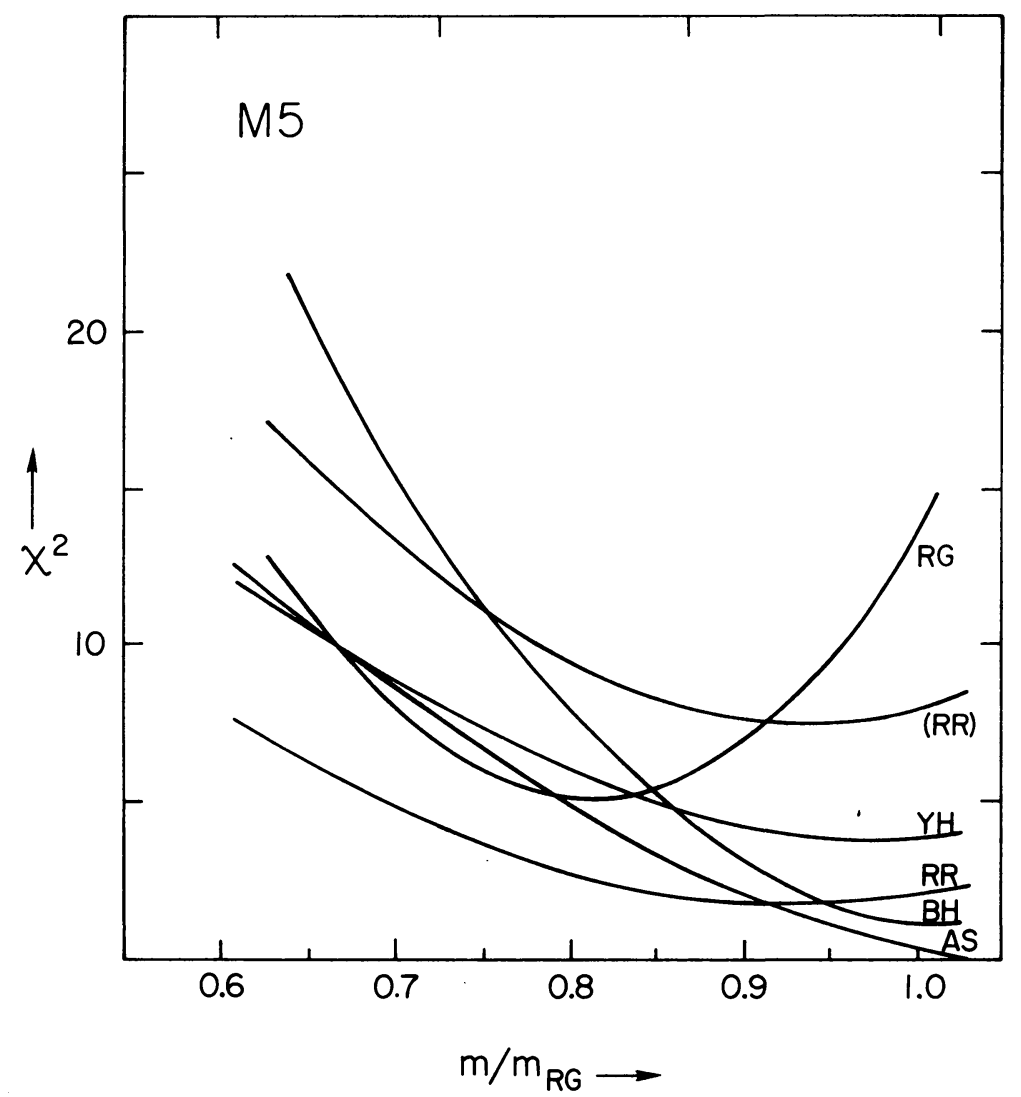

Fig. 7. Goodness-of-fit for observed distributions of stars of various types in M5, when compared with theoretical distributions of stars of various mass. Abbreviations stand for red giant, asymptotic branch, yellow horizontal branch, blue horizontal branch, and RR Lyrae. Data from Simoda and Tanikawa (1970, Table 3), except for (RR), which is from Oort and van Herk (1959, Table 15). Latter has 3 degrees of freedom; other distributions have 2. Red-giant star-count distribution disagrees with model, which was fitted to a surface-brightness distribution that is dominated by red giants. The two versions of the RR Lyrae distribution disagree with each other. 


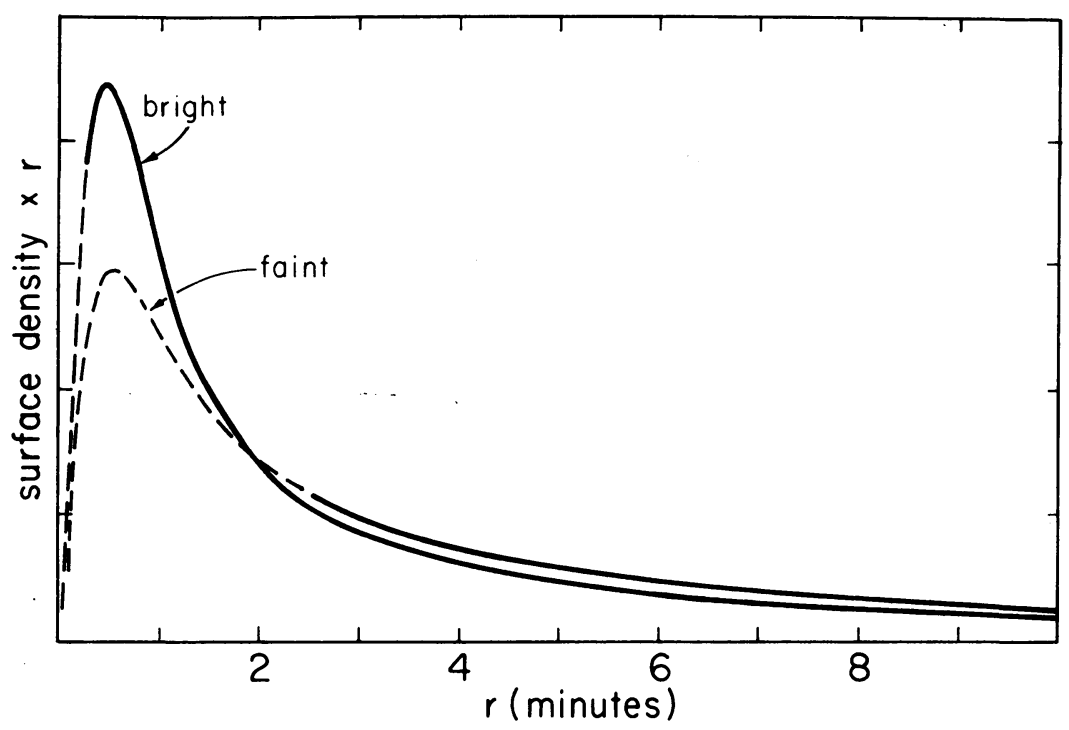

Fig. 8. Star numbers in $\mathbf{M} 3$, plotted in such a way that area under the curves is proportional to total star numbers. Dashed portions are unobservable, with extrapolation provided by dynamical model.

functions of different globular clusters disappear. It is interesting to note that the largest remaining uncertainty is due to not knowing the degree of anisotropy that should be put into the velocity distribution.

Another important application of cluster models is in determining the total masses of clusters from their observed internal velocity dispersions (Illingworth, 1975). It turns out, fortunately, that the observations allow a fairly direct determination of the central density in a cluster (see the discussion of elliptical galaxies, below); but to extrapolate from the central value to a total requires a model in which the stars of different mass have the correct relative distribution. It is interesting to note that observations of this sort give us our only information about the number of low-mass stars in globular clusters. The stars that we can see, which contribute practically all of the light, go only a little below the main-sequence turnoff; the less-massive stars make themselves known only by their gravitation.

Along with the ways in which observation works hand in hand with theory, we should also take note of cases in which star clusters have not been observed to behave in the way predicted by theory. The most striking of these is the case of the runaway centers. Numerical simulations have repeatedly shown cluster cores that become denser and denser, producing a central singularity at a finite point in time. Many clusters are old enough, dynamically, that they should already have reached this stage of central catastrophe, yet nowhere do we find either a central collapse going on, or else evidence that it has happened at some time in the past. Globular clusters have smooth central distributions, typically with a core radius of the order of a parsec, and there is no sign of additional central peaks. If as much as 100 solar masses of 
additional material were placed at the center of a high-concentration cluster of $10^{5}$ solar masses, its gravitation would perturb the distribution of the observed stars in a noticeable way. I know of only one cluster in which such a central excess might possibly exist. The central region of M15 shows a small excess of brightness over the profile that an isothermal core would give. The excess may or may not be observationally significant, but it certainly deserves closer examination.

One might imagine that open clusters are an even more likely place to look for central runaways, since their relaxation times are even shorter. For these poorer systems, however, it may be that the evolution of the small number of stars in the core soon becomes dominated by the massive central binary that always seems to form in the $N$-body calculations. The 'runaway' then consists merely of a continued transfer of energy between the central binary and its surroundings. If we think naively about the time scales, then nearly every open cluster should already have developed a massive central binary. A more realistic picture, however, includes stellar evolution; since the most massive stars have the shortest lifetimes, will they last long enough to play their assigned dynamical roles - and if not, how do the dynamic predictions change? Apparently, more theoretical work is needed on this problem. On the observational side, however, I do not know of any study at all in which open clusters are systematically searched for the present of a central, massive binary.

The presence of less spectacular binaries in clusters also poses an interesting observational problem. In a globular cluster, for instance, where the most massive stars have masses characteristic of the middle main sequence, W Ursae Majoris binaries should be the most massive stars in the cluster, and they should therefore concentrate strongly to the cluster center. Since they are far from being the brightest stars, these binaries will be very hard to detect; only in globular clusters of the lowest concentration can we hope to see individual stars of such low luminosity in the central regions. Unfortunately, the clusters of low concentration tend to be poor systems, with few stars of all kinds; and only a very few rich clusters have centers that are open enough for the search to be made. I do not know of any case where it has been done.

\section{Stellar Systems of Other Types}

Open clusters suffer, relative to globular clusters, from a smaller star number and a richer stellar background. Nevertheless they offer us several unique opportunities in supporting our dynamical theories with observational facts. Because they are younger in years than the globulars, open clusters still contain stars with a much wider range in mass. Furthermore, in many open clusters we can observe the distribution of the stars down to a lower limit of absolute magnitude than we can reach in any globular cluster. In clusters where we can use proper motions to separate faint cluster stars from the field, the magnitude range is especially large. Thus the nearby Hyades, although poor in stars, is in a sense the best-studied of all clusters (Pels et al., 1975).

Although younger in years, open clusters are older dynamically than are globular clusters, because their relaxation times are so much shorter. Most of the open clusters 
that were ever born are already dead, for dynamical reasons (Oort, 1958); and a statistical study of the ages and sizes of existing clusters gives us a check on their dynamical mortality (Wielen, 1971).

But from the observational point of view, perhaps the greatest value of open clusters is their very poorness in stars. These systems fall in the range of $N$ where our theoretical dynamics is very weak. For stellar encounters, these are the systems where large velocity changes are of comparable overall importance to small changes; for overall dynamics, these are the systems where the relaxation time is hardly longer than the crossing time. For both these situations (which are of course dynamically related), we lack a good theoretical treatment. Perhaps this is an area in which observations can guide a fledgling theory. As a starting point, one extensive set of star counts already exists (van den Bergh and Sher, 1960).

Elliptical galaxies lie at the opposite extreme, dynamically, from open clusters. They have so many stars that there is no doubt about the validity of large- $N$ representations, but they are less than one relaxation time old. What dynamical process determines their structure? The first step in answering this question is to examine the profiles of some actual systems; two of these are shown in Figures 9 and 10. In each case the curve that is fitted, more or less well, is that of a relaxed star-cluster model. Since the model has a nearly-Gaussian distribution of stellar velocities, the fit shows that elliptical galaxies have an internal velocity distribution that is nearly Gaussian. This should be a consequence, of course, of the violent relaxation (Lynden-Bell, 1967) that took place at the time when the galaxy formed.

Violent relaxation leads to a result that differs from that of stellar encounters in one

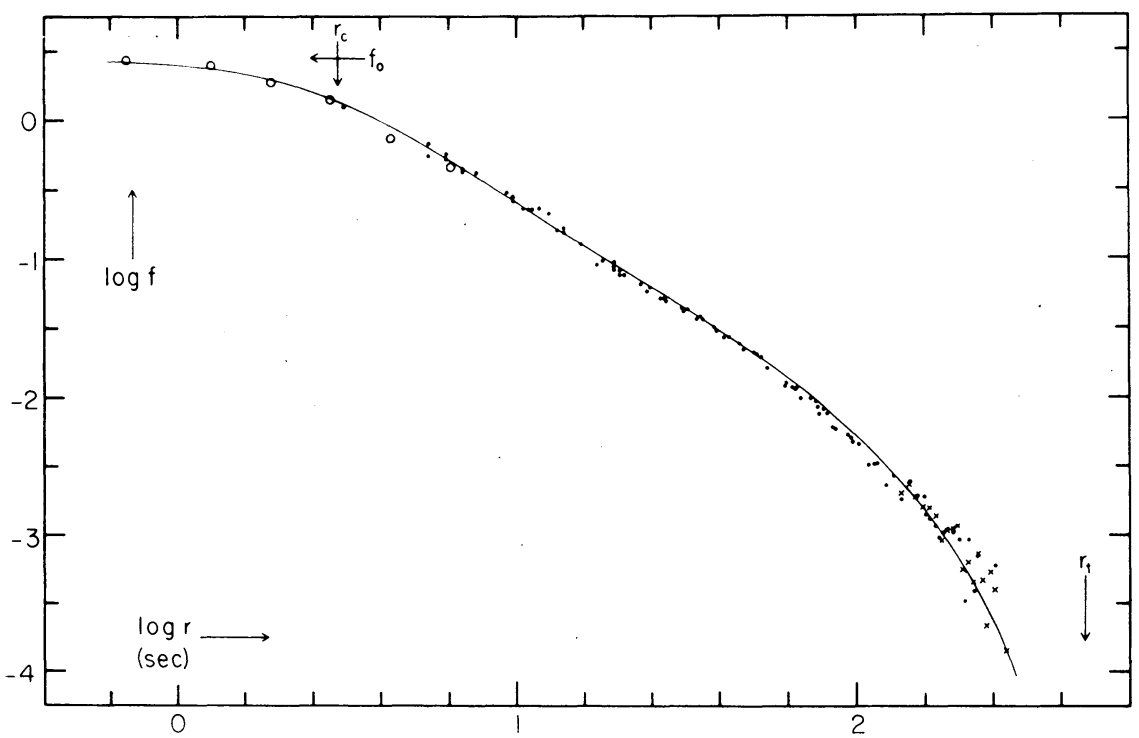

Fig. 9. Surface brightnesses in the elliptical galaxy NGC 3379, from data by Miller and Prendergast (1962). Curve is a star-cluster model. 


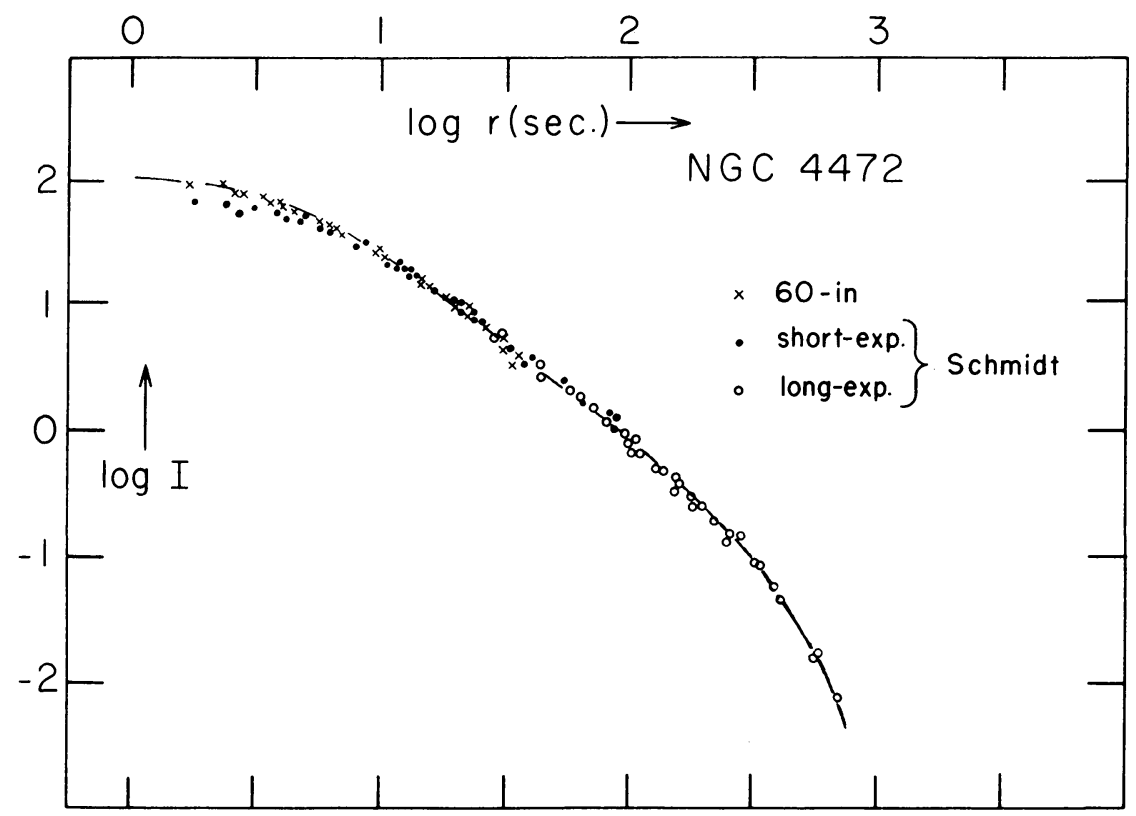

Fig. 10. Photographic surface brightnesses in the elliptical galaxy NGC 4472 , determined by the author. Same curve as used in Figure 9.

important way: it does not produce equipartition between stars of different mass. It is easily verified that equipartition does not exist in elliptical galaxies; if it did, then the envelope would consist almost completely of red dwarf stars, and its color would be a deep and unmistakable red.

The profile of elliptical-galaxy envelopes poses a problem, however, which should not be obscured by the apparent ease of fitting star-cluster models to them. The theoretical curve used in fitting the galaxies in Figures 9 and 10 has an envelope shape that is determined by the particular ratio of the tidal cutoff radius to the core radius of the system, but this cannot possibly be the mechanism that molds the envelopes of elliptical galaxies. As Hubble showed long ago (Hubble, 1930), all elliptical galaxies have profiles that resemble these, with the surface density falling approximately as $r^{-2}$ (space density as $r^{-3}$ ). It is inconceivable that every elliptical galaxy could have a tidal cutoff distance that is always the same multiple of its core radius.

What then has determined the profile of the envelopes of elliptical galaxies? Since relaxation has not changed these systems, we must be seeing a result of the process that originally formed them. Specifically, the profile of the envelope is determined by the particular way in which the high-energy tail of the velocity distribution drops to zero, and this is what we should examine.

There is more to elliptical galaxies than a mere radial profile; they are, after all, elliptical rather than spherical. Rotation obviously plays an important role in determining their forms. Theoretical models have been developed by Wilson (1975a, b); they fit the observed isophote shapes to a large extent, but there are some isophotal 
profiles that Wilson's models are unable to fit. The greatest observational lack is detailed information about the rotations of elliptical galaxies. Do the systems with different isophotal profiles also have different patterns of rotation ? We would like very much to know.

An application for which it is important to have a good dynamical picture of elliptical galaxies is the determination of their masses. From an observation of the internal velocity dispersion and the relative distribution of density, it is possible in principle to determine the mass of the system. One might imagine naively that all that is needed is to apply the virial theorem. Such an approach can unfortunately produce very bad results, since it invariably involves serious and uncertain extrapolations. (For a detailed discussion, see Rood et al., 1972, Section VIII.)

In fact, the quantity that should be determined from the observations is the quantity that really does follow directly from them: the central density. In practice, the only place at which we know the velocity dispersion in an elliptical galaxy is in its bright central region; hence this is the only place in which we can determine masses. Since observations give us the central distribution of light, we can also derive a central $M / L$, which is one of our intrinsic objectives in any case. But all else is extrapolation.

It should be no surprise that clusters of galaxies have density profiles like those inside of single elliptical galaxies. They also have relaxation times longer than their ages but were subjected to violent relaxation at the time of their formation. Figure 11 shows the distribution of galaxies in the Coma cluster, fitted with the same curve that was used for the elliptical galaxies in Figures 9 and 10. The detailed problems may turn out to be very different, however. Even though we still have relatively little

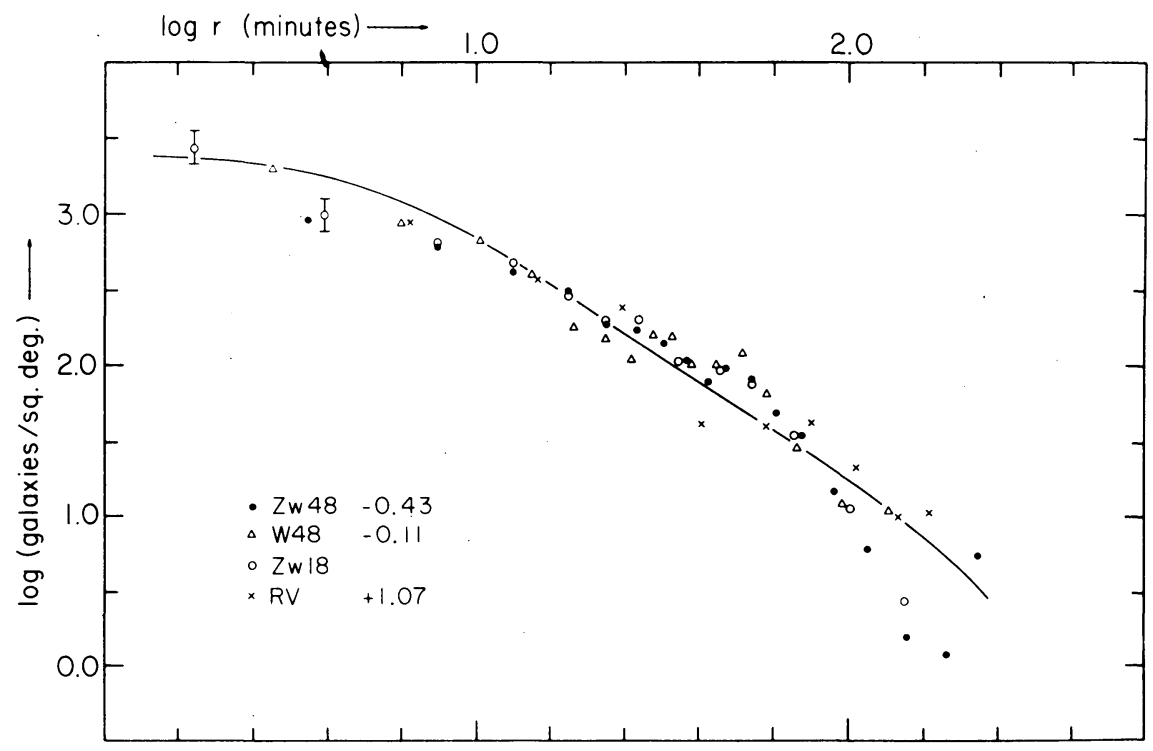

Fig. 11. Surface densities of galaxies in the Coma cluster, as collected from various sources and fitted together by Rood et al. (1972). Same curve as used in Figures 9 and 10. 
information about clusters of galaxies, it is clear that new problems arise - for example, the clusters whose centers contain a single, dominant supergiant galaxy. This is usually a $\mathrm{cD}$ galaxy, whose profile appears to be different from that of a 'normal' elliptical. What are these galaxies? It is likely that the first steps toward answering such questions will be observational.

\section{Conclusion}

In this review I have tried to emphasize the importance that observations should have for a right-thinking theoretician. The nature of the Universe may eventually be rationalized by theoretical understanding, but it will not be discovered in the first place by pure thought. Any theory rests upon idealizations, and we should be sure that they are the correct ones. This admonition applies equally to simulations, where there is an equal danger of solving the wrong problem.

At the same time, I have tried to indicate directions in which theory indicates that observation should go. Our future progress will depend on a well-chosen combination of our various techniques.

\section{Acknowledgements}

The preparation of this paper was supported by NSF Grant GP-40483X.

\section{References}

Griffin, R. F.: 1972, Observatory 92, 28.

Gunn, J. E. and Griffin, R. F.: 1971, in Ann. Report Dir. Hale Obs., p. 411.

Hodge, P. W.: 1966, Ann. Rev. Astron. Astrophys. 9, 35.

Hubble, E.: 1930, Astrophys. J. 71, 231.

Illingworth, G. D. : 1975, this volume, p. 151.

King, I. R. : 1962, Astron. J. 67, 471.

King, I. R.: 1966a, Astron. J. 71, 64.

King, I. R. : 1966b, Astron. J. 71, 276.

King, I. R.: 1972, Astrophys. J. 174, L123.

King, I. R., Hedemann, E., Hodge, S. M., and White, R. E.: 1968, Astron. J. 73, 456.

Lynden-Bell, D.: 1967, Monthly Notices Roy. Astron. Soc. 136, 101.

Michie, R. W. and Bodenheimer, P. H.: 1963, Monthly Notices Roy. Astron. Soc. 126, 269.

Miller, R. H. and Prendergast, K. H.: 1962, Astrophys. J. 136, 713.

Oort, J. H. : 1958, in D. J. K. O'Connell (ed.), Stellar Populations, North Holland Press, Amsterdam, p. 510.

Oort, J. H. and van Herk, G. : 1959, Bull. Astron. Inst. Neth. 14, 299 (No. 491).

Pels, G., Oort, J. H., and Pels-Kluyver, H. A.: 1975, this volume, p. 159.

Peterson, C. J.: 1974, Astrophys. J. 190, L17.

Peterson, C. J. and King, I. R.: 1975, Astron. J. 80 (in press).

Rood, H. J., Page, T. L., Kintner, E. C., and King, I. R.: 1972, Astrophys. J. 175, 627.

Simoda, M. and Tanikawa, K.: 1970, Publ. Astron. Soc. Japan 72, 143.

Spitzer, L. and Härm, R.: 1958, Astrophys. J. 127, 544.

van den Bergh, S., and Sher, D.: 1960, Publ. David Dunlap Obs., Vol. II, No. 7.

Wielen, R.: 1971, Astron. Astrophys. 13, 309.

Wielen, R.: 1975, this volume, p. 119.

Wilson, C. P.: 1975a, Astron. J. 80, 175.

Wilson, C. P.: 1975b, this volume, p. 207.

Wilson, C. P. and King, I. R. : 1975, Astron. J. 80 (in press). 


\section{DISCUSSION}

Miller: A warning to the theoreticians here - although King has stressed the extent to which observations delimit theories, there is still a good deal of prejudice in the analytic forms used. This is common to all such attempts (if anything, King is more candid than most in admitting to such prejudices). If the problem is turned around, and regarded as a problem in statistical inference on some parameters, it usually turns out that the parameters are remarkably poorly determined. With allowance for backgrounds, King's models allow 4 adjustable parameters, and should surely give a good fit to any reasonable set of observations. I've tried some cluster fits to a Plummer model plus background, and gotten remarkable good fits ( 3 parameters), but then tried with another analytic form (also 3 parameters) and found an equally good fit. In fact, the fits were too good.

King: I admit the prejudice freely. It is a question of philosophy: I am quite happy to accept an agreement of observations with a theoretical picture that is a priori highly plausible. As for backgrounds, I do not think that it is at all correct to call the background a fourth parameter. The background level is indeed determined observationally, but these observations are quite separate from the ones used to determine the other three parameters. Typically we counted an external surrounding region larger than the cluster itself, so that the statistical error in the background determination is negligibly small. It is very unlikely that the background levels have systematic errors either; we checked for this by counting entire fields that did not have a cluster in then, and then testing for uniformity. Finally, I suspect that your success in fitting cluster data with a Plummer model was due to using data with large statistical uncertainties. I don't think that you could fit good-quality globular-cluster data with a Plummer model at all.

Brosche: If three parameters are sufficient for the description of globular clusters and elliptical galaxies, are they necessary as well, in other words, does any relation exist between those parameters?

King: Peterson has looked at all sorts of correlations between quantities, and the only one that seems to hold up consistently is the correlation between observed limiting radius and calculated limiting radius. Otherwise there seem to be no significant correlations.

Lecar: How do you determine the tidal radius as related to the orbit of the globular cluster? Do you use current position or peri-galacticon?

King: In the diagram that I showed, the observed radii were determined from observation, by fitting standard curves. The 'calculated' values were for the cluster's present position. Thus the ratio of the two should be an indication of the ratio between the cluster's present distance from the Galactic Center and its perigalactic distance.

Kalnajs: I presume that color variations across clusters do not give any useful information about mass-segregation. Can you tell us why not?

King: The color changes to be expected are quite small - a couple of hundredths of a magnitude in $B-V$. When you look at the statistical uncertainties in the colors of the regions that have to be measured, because of the small number of stars that contribute most of the light, the uncertainties are as large as the quantities that you are trying to measure. 